\title{
Initial steps on the synthesis of new antimicrobial fumiquinazoline related alkaloids
}

\author{
Mariana C. Almeida ${ }^{1,2}$, Diana I. S. P. Resende ${ }^{1,2^{*}}$, Paulo Costa ${ }^{2,3}$, Madalena Pinto ${ }^{1,2^{*}}$, Emília Sousa ${ }^{1,2}$ \\ ${ }^{1}$ Laboratório de Química Orgânica e Farmacêutica, Faculdade de Farmácia, Universidade do Porto, Rua de Jorge Viterbo Ferreira 228, 4050-313 Porto, Portugal; \\ ${ }^{2}$ CIIMAR - Centro Interdisciplinar de Investigação Marinha e Ambiental, Terminal de Cruzeiros do Porto de Leixões, 4450-208 Matosinhos, Portugal \\ ${ }^{3}$ ICBAS - Instituto de Ciências Biomédicas Abel Salazar, Universidade do Porto, Rua de Jorge Viterbo Ferreira 228, 4050-313 Porto, Portugal
}

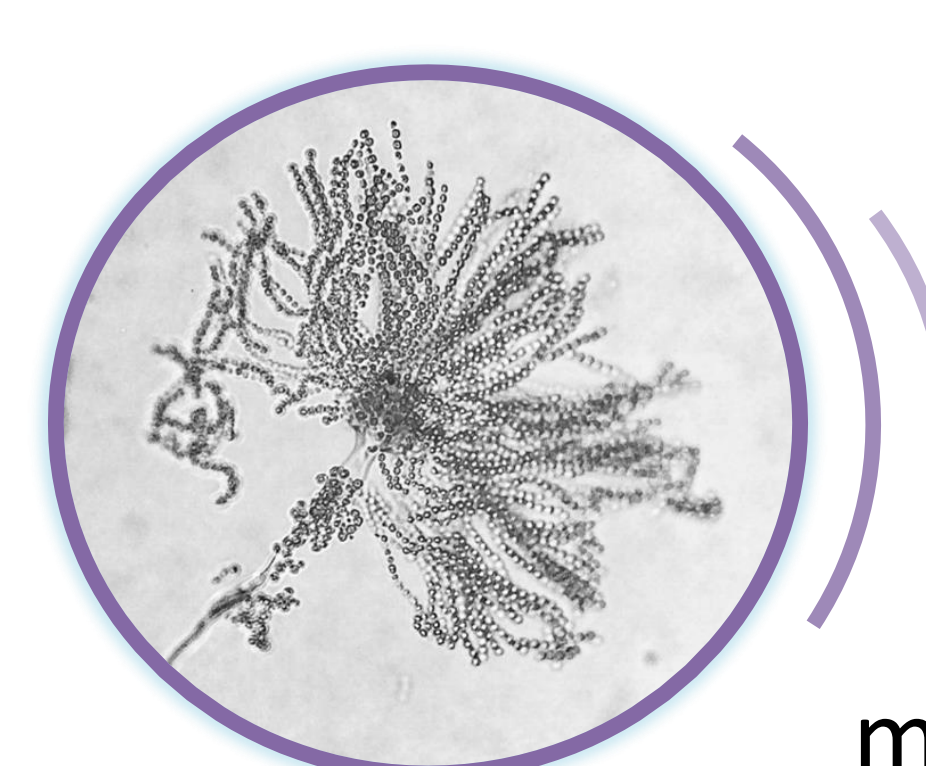

Currently drug resistance is rising to dangerously high levels worldwide and threatening our ability to treat even common infectious diseases [1]. Secondary metabolites, especially alkaloids containing an indole group and structurally related to fumiquinazolines, can be found in both marine and terrestrial secondary metabolites and are of great importance in the area of drug discovery with promising antimicrobial properties [2].

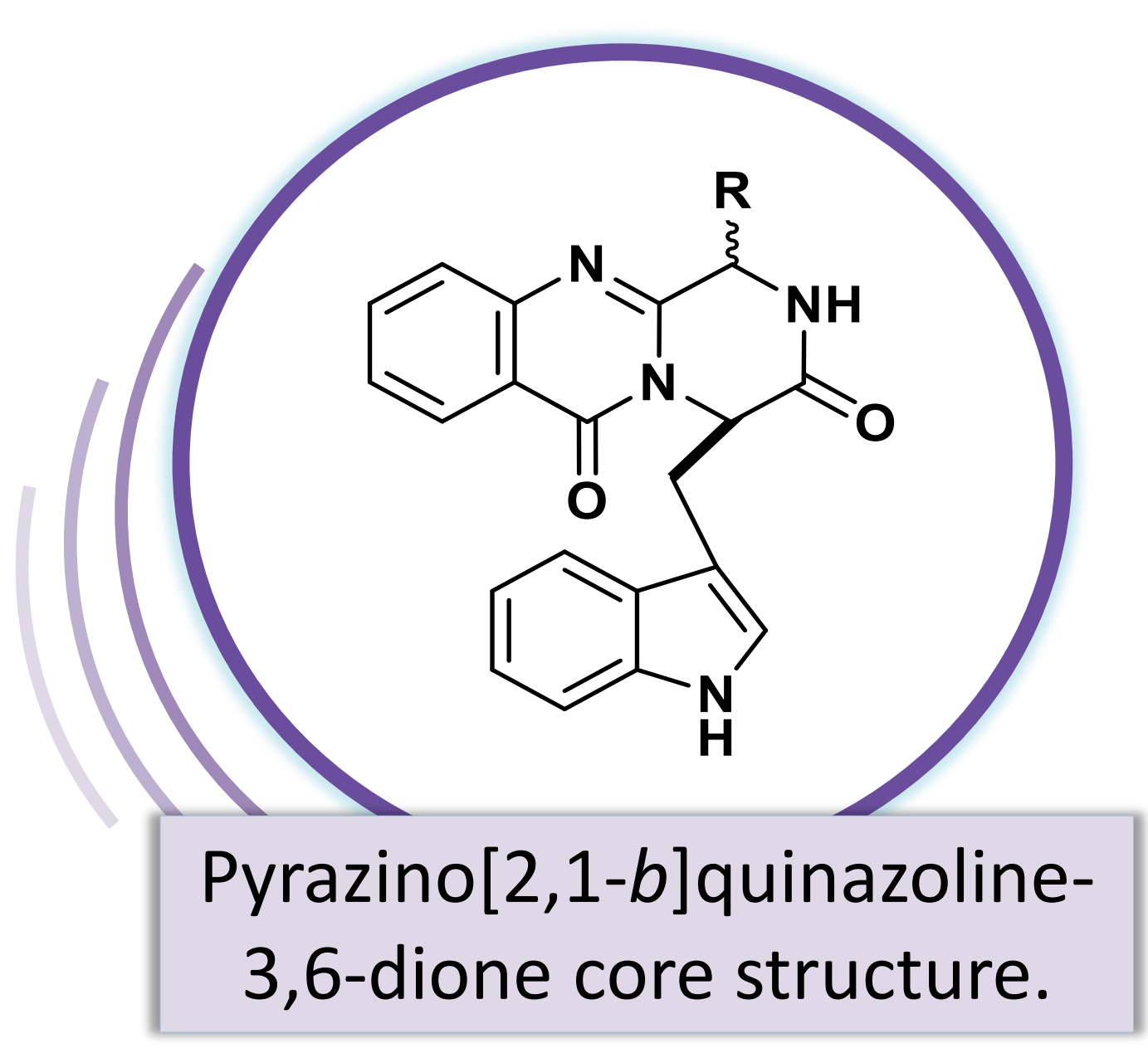

This work aims to synthesize alkaloids related to fumiquinazolines, as well as introducing new molecular modifications, in order to improve their properties as antimicrobial agents.

\section{Results}

A multi-step synthetic pathway [3] was followed through conjugation of anthranilic acid, D-tryptophan methyl ester hydrochloride and a third amino acid (glycine or alanine).

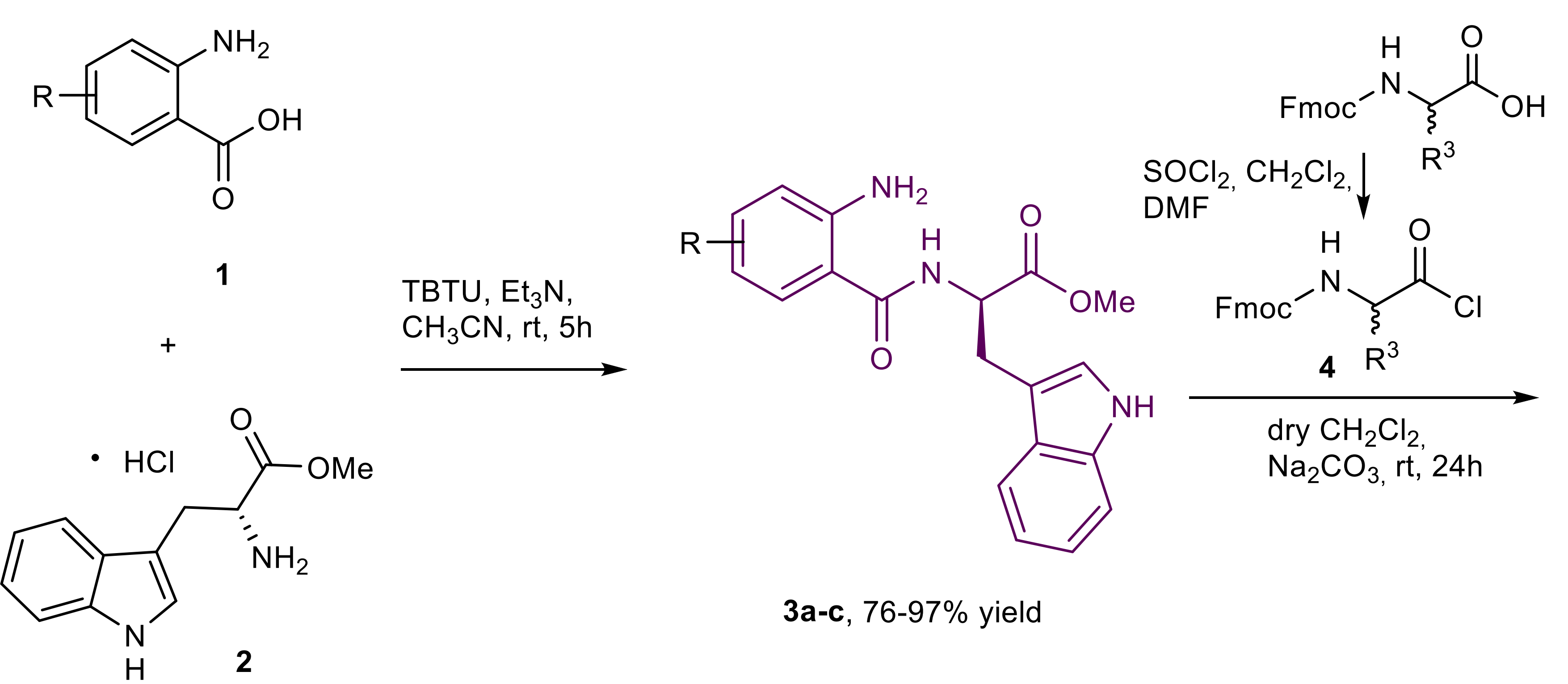

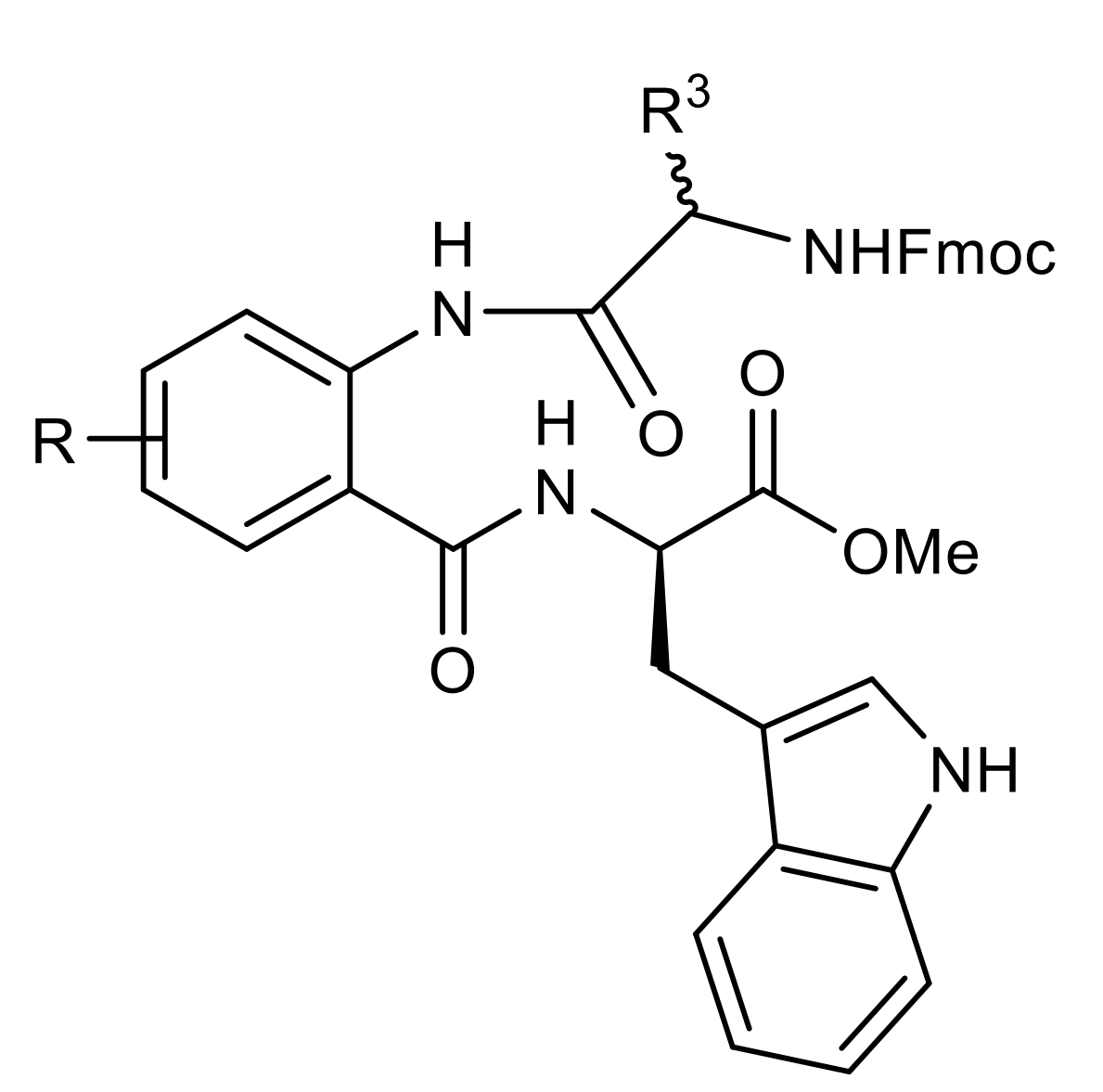

5a-d, in progress

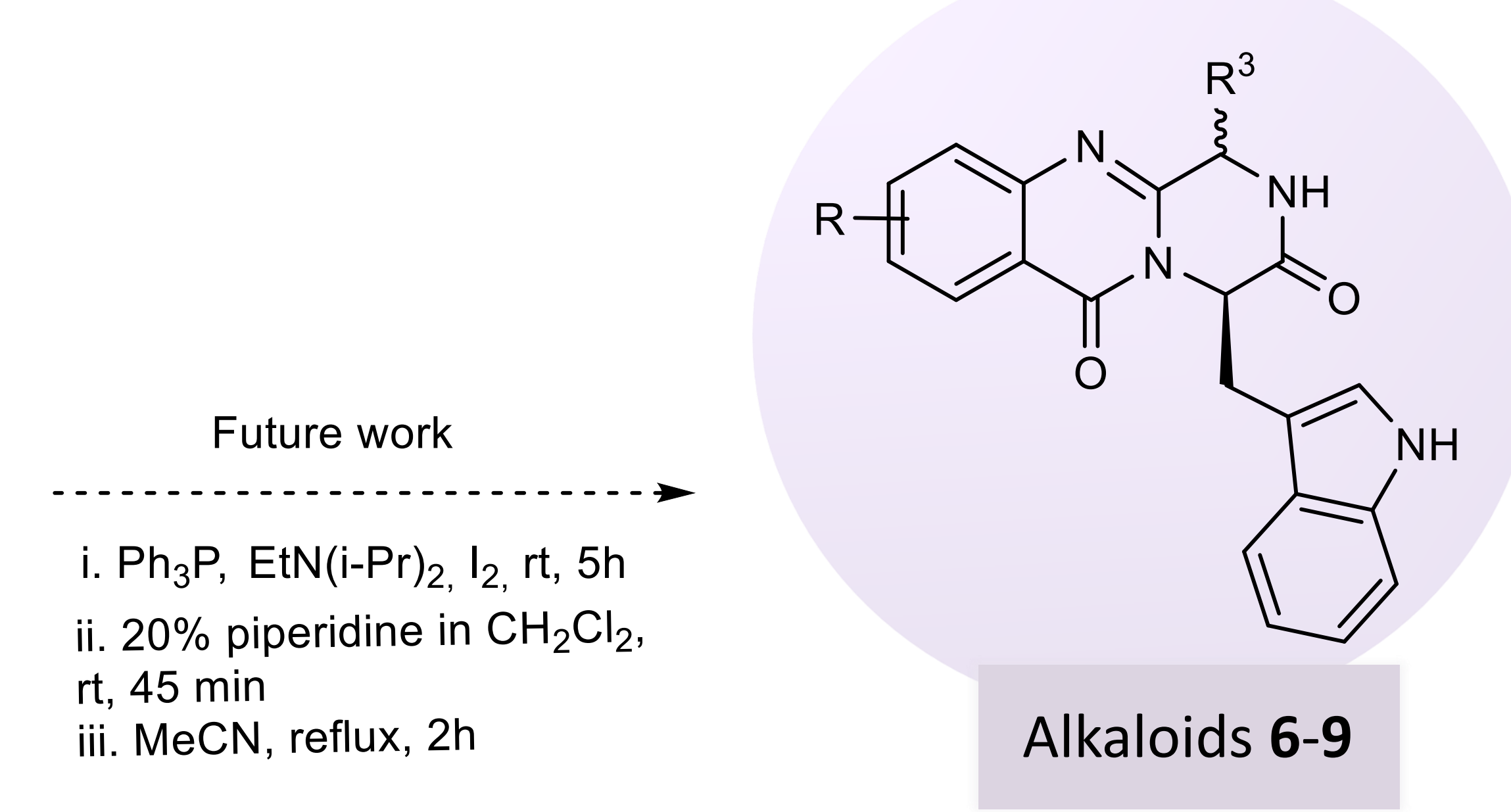

Alkaloids 6-9

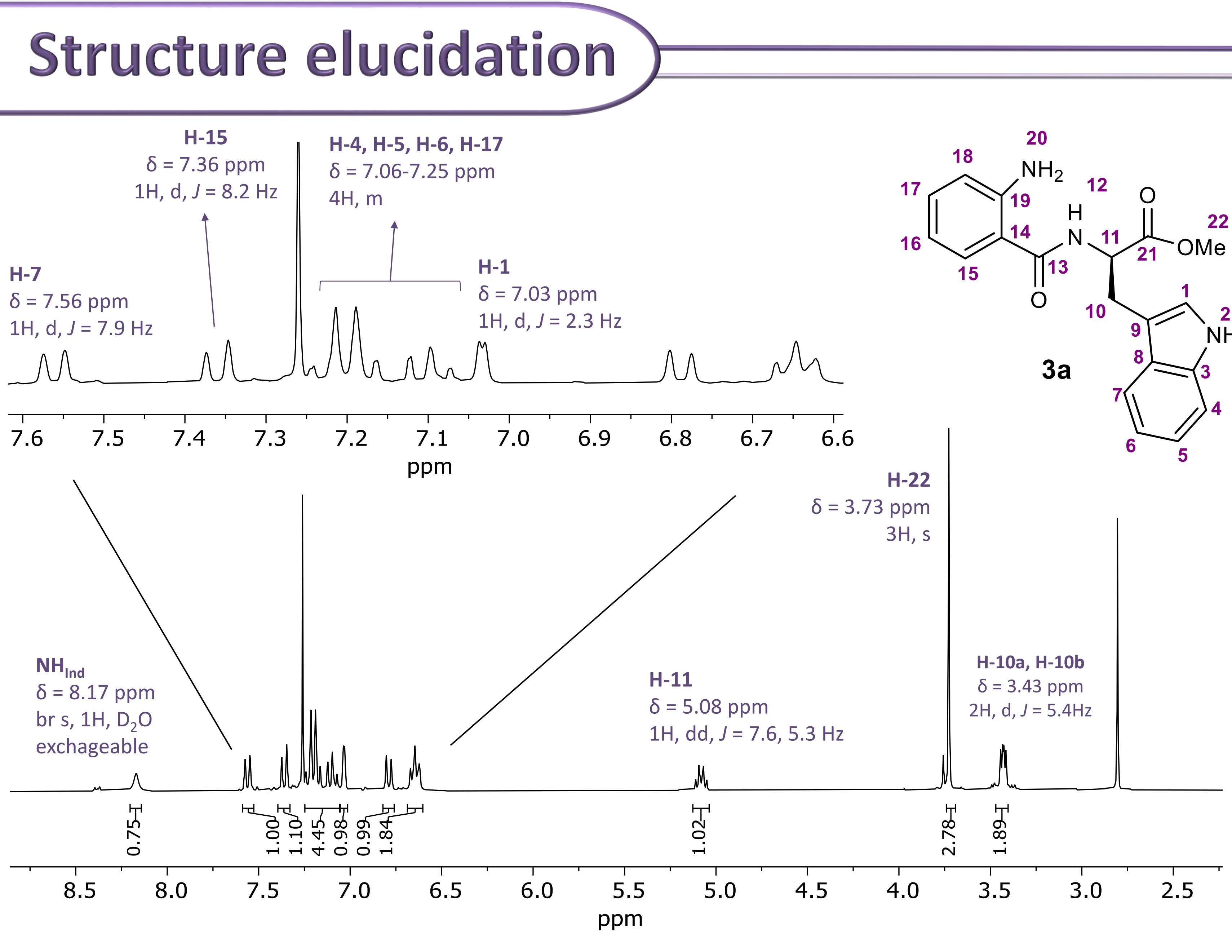

Figure 1. ${ }^{1} \mathrm{H} N \mathrm{NMR}\left(\mathrm{CDCl}_{3}\right)$ of $\mathrm{N}$-(2-aminobenzoyl)-D-tryptophan methyl ester (3a).

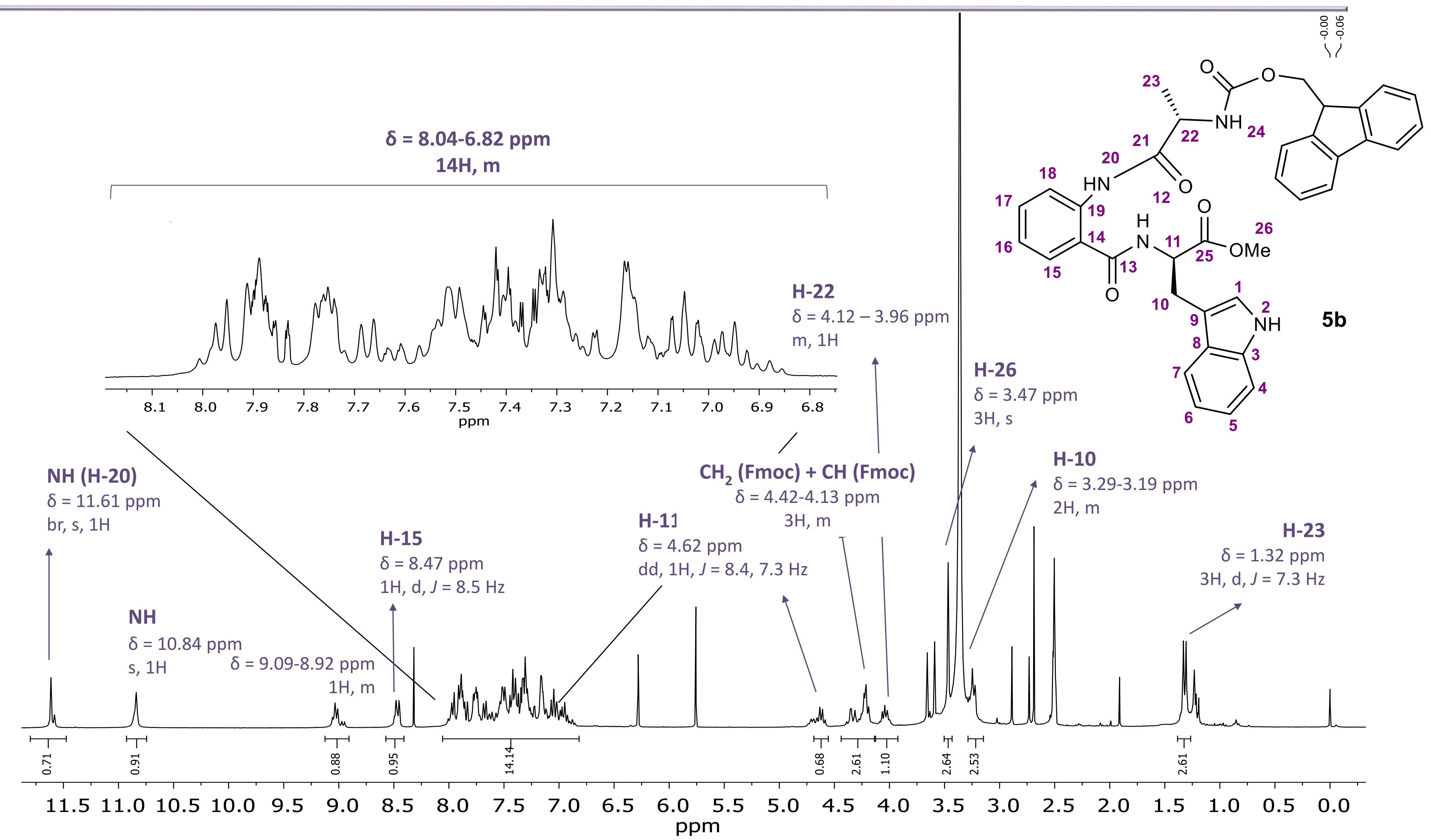

Figure 2. ${ }^{1} \mathrm{H}-\mathrm{NMR}(\mathrm{DMSO})$ of methyl $(R)-2-(2-((R)-2-((((9 H-$-fluoren-9-yl)methoxy)carbonyl) amino)propanamido)benzamido)-3-(1H-inden-3-yl)propanoate (5b).

\section{Conclusions}

- Linear dipeptide 3 and tripeptide 5 alkaloid intermediates were synthesized to be further used towards the synthesis of alkaloids 6-9, structurally related to fumiquinazolines.

- Structure elucidation by ${ }^{1} \mathrm{H}-\mathrm{NMR}$ spectroscopy indicated that the obtained compounds correspond to the expected structures.

- Future work will involve screening of the new alkaloids for their antibacterial activities.

\section{References}

1. Cheng, G. et al. Frontiers in Microbiology 2016, 7, 470; 2. Resende, D., et al. Nat. Prod. Rep., 2019, 36, 7-34; 3. Wang, H., et al. J. Org. Chem. 2000, 65, 1022-1030.

Acknowledgments

This research was supported by national funds through FCT - Foundation for Science and Technology within the scope of UIDB/04423/2020, UIDP/04423/2020 (Group of Natural Products and Medicinal Chemistry, CIIMAR), and under the project PTDC/SAU-PUB/28736/2017 (reference POCI-01-0145-FEDER-028736), co-financed by COMPETE 2020, Portugal 2020 and the European Union through the ERDF and by FCT through national funds, as well as CHIRALBIOACTIVE-PI-3RLIINFACTS-2019. Mariana C. Almeida acknowledges the BYT+ scholarship to CIIMAR.

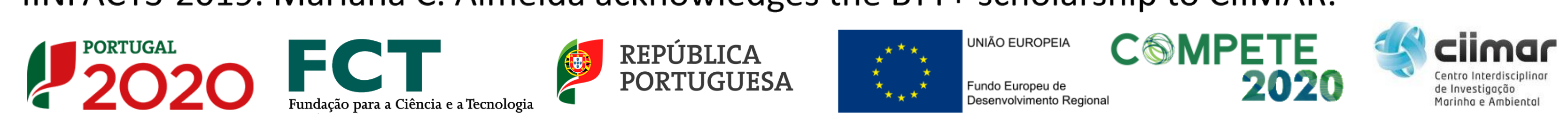

